\title{
ENO2 Promotes Cell Proliferation, Glycolysis, and Glucocorticoid-Resistance in Acute Lymphoblastic Leukemia
}

\author{
Cheng-cheng Liu ${ }^{a, b, c}$ Hua Wang ${ }^{a, b, c}$ Wei-da Wanga, ${ }^{a, b, c}$ Liang Wang ${ }^{a, b, c}$ \\ Wen-jian Liu ${ }^{a, b, c}$ Jing-hua Wang ${ }^{a, b, c}$ Qi-rong Geng ${ }^{a, b, c}$ Yue Lu $u^{a, b, c}$
}

a'Department of Hematologic Oncology. Sun Yat-sen University Cancer Center. Guangzhou, Guangdong, bState Key Laboratory of Oncology in South China. Guangzhou, Guangdong, 'Collaborative Innovation Center for Cancer Medicine. Guangzhou, Guangdong, P.R. China

\section{Key Words}

Acute lymphoblastic leukemia • Enolase 2 - Biological marker • Glycolysis • Glucocorticoid resistance

\begin{abstract}
Background/Aims: The metabolic features of cancer cells have long been acknowledged to be altered and to provide new therapeutic opportunities. The expression of glycolytic enzyme enolase 2 (ENO2) was found to be closely associated with the clinical features of acute lymphoblastic leukemia (ALL) patients, but its functions remain unclear in ALL. Methods: We evaluated the association between ENO2 mRNA expression in bone marrow mononuclear cells (BM-MNCs) and the efficacy of chemotherapy, and further explored the function of ENO2 in ALL. The molecular mechanisms of ENO2 expression and its effects on cell growth, glycolysis and glucocorticoid resistance were explored by Cell Counting Kit-8, glucose-consumption assay, Quantitative RT-PCR, Western blotting and in vivo tumorigenesis in NOD/SCID mice. Results: The results showed that ENO2 mRNA expression in BM-MNCs was significantly decreased when patients completed induction chemotherapy and reached complete remission (CR). ENO2 mRNA expression was increased when patients suffered relapse. Functional studies demonstrated that ENO2 promoted cell growth, glycolysis, and glucocorticoid resistance, all of which were effectively inhibited when ENO2 was silenced with shRNAs. Further studies revealed that ENO2 up-regulated various glycolysis-related genes and enhanced Akt activity with subsequent glycogen synthase kinase $3 \beta$ (GSK-3 $\beta$ ) phosphorylation, inducing cell proliferation and glycolysis. The combination of silencing ENO2 and 2-deoxyglucose (2-DG) synergistically inhibited leukemia cell survival. Conclusions: These results indicate that ENO2 may be a biological marker for monitoring chemotherapeutic efficacy and relapse in ALL. ENO2 may provide a potential therapeutic strategy for ALL.
\end{abstract}

C.-C. Liu and H. Wang contributed equally to this work.

Yue Lu

KARGER
Department of Hematologic Oncology. Sun Yat-sen University Cancer Center. Guangzhou, Guangdong, State Key Laboratory of Oncology in South China. Guangzhou, Guangdong (P.R. China); E-Mail lvyue@sysucc.org.cn 


\section{Introduction}

Acute lymphoblastic leukemia (ALL) is a heterogeneous disease, both in terms of its pathology and the populations that it affects. Although the treatment of children with ALL has achieved great success, the results of adult ALL are lagging behind [1]. Adults with ALL at high risk in diagnosis are more likely to relapse and show resistance to chemotherapy after achieving initial complete remission (CR) [2-4]. In the past decade, the treatment of ALL has made significant progress in understanding the pathogenesis of disease, predicting prognosis, and developing new therapies. The application of targeted therapy significantly improved the survival of patients with ALL [5-8]. Further improvements in the outcome of ALL therapy require the development of novel, targeted and low toxic therapies. Additional biomarkers are required to further optimize stratification and guide the treatment of this disease.

Enolase 2 (ENO2), also known as neuron-specific enolase (NSE), which includes two enolase isoenzymes, $\gamma \gamma$ and $\alpha \gamma$, is primarily expressed by mature neurons and cells of neuronal origin. ENO2 plays an important role in glycolysis, promoting the conversion of $\beta$-glycerophosphate into dihydroxyacetone phosphate $[9,10]$. In previous studies, we found that serum ENO2 was closely associated with several clinical features, such as immunophenotype, risk stratification and serum lactate dehydrogenase (LDH) levels in ALL. Multivariate analysis revealed that high ENO2 expression was an independent prognostic factor for adult ALL patients. However, there are no reports demonstrating the function of ENO2 in ALL. In the present study, we further explored the potential role of ENO2 as a therapeutic target in ALL.

\section{Materials and Methods}

\section{Patients, bone marrow samples and cell lines}

Fifteen newly diagnosed ALL adult patients treated at the Department of Hematologic Oncology, Cancer Center, Sun Yat-Sen University were included in the present study. Bone marrow samples were collected at initial diagnosis, the end of induction therapy, after complete remission and at relapse. Normal bone marrow samples were obtained from individuals suspected as patients with hematologic malignancy but were actually normal. Mononuclear cells were isolated by Lymphoprep density gradient centrifugation. Informed consent was obtained from the patients prior to their participation in the present study. The Reh, Jurkat, Molt-4 and CCREF-CEM cell lines (ATCC, Manassas, VA, USA) were cultured in RPMI-1640 medium (Gibco, Carlsbad, CA, USA) supplemented with 10\% fetal bovine serum (FBS), $1 \% \mathrm{~L}$-glutamine, $100 \mathrm{U} / \mathrm{mL}$ penicillin and $100 \mu \mathrm{g} / \mathrm{mL}$ of streptomycin under standard culture conditions $\left(37^{\circ} \mathrm{C}\right.$ in $\left.5 \% \mathrm{CO}_{2}\right)$. HEK293T, a human embryonic kidney cell line, was used for the production of viral particles. Cell viability and cell count were determined by a trypan blue exclusion-staining assay and analysed by MACSQuant.

\section{Lentiviral production and infection}

High-titre lentiviral vector stocks were produced in HEK293T cells by liposomalmediated transfection of the modified transfer vector HBLV-CMVIE-ZsGreen-T2A-puro and the packaging vectors pSPAX2 and pMD2G. The viral particles were harvested and concentrated by ultracentrifugation. Reh and Jurkat cells were infected with recombinant virus particles in the presence of $6 \mu \mathrm{g} / \mathrm{ml}$ of polybrene (Sigma, St Louis, MO, USA). Cells transfected with empty vector were used as controls. After expansion and maintenance in RPMI-1640 medium supplemented with 10\% FBS for 5 days, the infected cells were positively selected for puromycin resistance.

Quantitative RT-PCR

RNA was extracted according to the manufacturer's protocol, and subsequently cDNA was synthesized. ENO2 mRNA levels were quantified based on the incorporation of SYBR KARGER 
Green (Thermo Scientific, Waltham, MA, USA) by quantitative real-time PCR. Quantitative RT-PCR was performed for each sample in duplicate with GAPDH as a housekeeping gene. The comparative cycle threshold $(\mathrm{Ct})$ method was used to determine the relative expression of ENO2. Each sample was tested in triplicate. The primers used for amplification of ENO2 were 5'-CGTTACTTAGGCAAAGGTGTCC-3' (forward) and 5'-CTCCAGCATCAGGTTGTCCAGT-3' (reverse). The primers used for the amplification of the reference gene GAPDH were 5'-AGGTCGGTGTGAACGGATTTG-3' (forward) and 5'-TGTAGACCATGTAGTTGAGGTCA$3^{\prime}$ (reverse).

\section{Protein isolation and Western blot analysis}

The cells were lysed using a nuclear and cytoplasmic protein extraction kit according to the manufacturer's instructions. The extracts were centrifuged at 12, $000 \mathrm{rpm}$ for $15 \mathrm{~min}$ at $4^{\circ} \mathrm{C}$, and the supernatant was collected. A BCA protein assay kit (Pierce, Hercules, CA, USA) was used to determine the protein concentrations. Protein (40 mg) was loaded on Bio-Rad Mini-Protean gels and transferred onto polyvinylidene fluoride membranes. The membranes were blocked with 5\% skim milk and probed with 1:1000 anti-ENO2 (Abcam, Cambridge, MA, USA) and anti-GAPDH 1:20000 (Abcam, Cambridge, MA, USA) in 5\% skim milk, followed by anti-rabbit secondary antibodies (Beyotime Biotechnology, Shanghai, China). The proteins were detected with the respective antibodies by using an ECL kit (Pierce, Hercules, CA, USA).

\section{Glucose-consumption assay}

The cells were cultured in complete medium for 24 hours. The medium of each group was respectively collected at $6,12,18$, and $24 \mathrm{~h}$. The glucose concentration of each sample was analysed by using colorimetric kits according to the manufacturer's instructions (Biovision, Mountain View, CA, USA). The absorbance was measured at $450 \mathrm{~nm}$ using a Multiskan MK3 microplate-reader (Thermo Labsystem).

\section{Cell viability}

Cell viability was detected by Cell Counting Kit-8 (CCK8, Tong Ren, Japan). The cells were placed onto 96-well plates at a concentration of $1 \times 10^{4}$ cells/well, and subjected to various treatments prior to analysis by the CCK-8 assay. The absorbance values in each well were measured using a microplate reader at $450 \mathrm{~nm}$. All experiments were performed three times.

\section{Animal experiment}

Female NOD/SCID mice (4-5 weeks old and weighing 17-23 g) were housed and maintained at the animal facility of the Laboratory Animals Centre of Sun Yat-sen University at $22^{\circ} \mathrm{C}$ and under a 12-hour light/dark cycle with free access to food and water. The mice were randomly assigned into four groups. Cells $\left(5 \times 10^{6}\right)$ were subcutaneously injected into the NOD/SCID mice. The volume was calculated as follows: volume $=(\pi \times$ length $\times$ width $\times$ heig ht) 6 . The tumor xenograft weights were also measured. All mice were humanely sacrificed by $\mathrm{CO} 2$ inhalation prior to death. All animal maintenance and procedures were performed in strict accordance with the recommendations established by the Animal Care and Ethics Committee of Sun Yat-sen University as well as the U.S. National Institutes of Health Guide for the Care and Use of Laboratory Animals. The protocol was approved by the Animal Care and Ethics Committee of Sun Yat-sen University. In animal studies, all efforts were made to minimize the suffering of mice.

\section{Statistical analysis}

Data are presented as the means \pm SD. Statistical analysis was calculated by one-way ANOVA with Dunnett's test as a post-test and the two-tailed unpaired t test using SPSS 16.0 software. P-values of $<0.05$ were considered statistically significant. 


\section{Results}

ENO2 expression in ALL patients and cell lines

To explore whether ENO2 mRNA levels vary at initial diagnosis and at complete remission after treatment, fifteen patients with ENO2positive expression who achieved CR were investigated, and eight of these fifteen patients later suffered from relapse. After induction chemotherapy, the ENO2 mRNA level was significantly decreased compared with that prior to treatment, and the ENO2 mRNA level was the lowest when CR was achieved $(\mathrm{P}<0.01$ Fig. 1A). However, when the patient relapsed, ENO2 mRNA levels were elevated $(\mathrm{P}<0.05$ Fig. $1 \mathrm{~A})$.

ENO2 protein was highly expressed in ALL cell lines compared to that in normal peripheral blood lymphocytes, and the ENO2 protein levels in the BMMNCs of three ALL patients were also significantly higher than those in the BM-MNCs of healthy controls (Fig. 1B). Further, ENO2 was expressed in both the cytoplasm and cytomembrane in Reh, Jurkat and CCRF-CEM cells by immunofluorescence assay (Fig. 1C).

\section{ENO2 enhances ALL cell growth}

To evaluate the functional significance of ENO2 in ALL, we silenced and overexpressed ENO2 in two different acute lymphoblastic leukemia cell lines (Reh and Jurkat). First, we established ENO2 silencing in Reh cells (designated as ENO2-sh1-Reh and ENO2-sh2Reh) and Jurkat cells (designated as ENO2-sh1-Jurkat and ENO2-sh2-Jurkat) by lentiviral transduction. Second, ENO2 overexpressing Reh cells (designated as ENO2-Reh) and Jurkat cells (designated as ENO2-Jurkat) were established. The ENO2 expression levels in these cell lines were verified by RT-PCR and Western blotting (Fig. 2A, 2B, 2C and 2D). Subsequently, these cell lines were used to examine the effects of ENO2 on cell growth by CCK-8 assays. We found that the growth of both ENO2-sh1 and ENO2-sh2 Reh cells was significantly slower than that of sh-Control Reh cells after 72 hours (Fig. 2E). ENO2-sh1 and ENO2-sh2 Jurkat cells also showed slower growth than sh-Control cells. In contrast, the overexpression of ENO2 in Reh and Jurkat cells significantly promoted cell growth compared to that of the control cells over time (Fig. 2E).

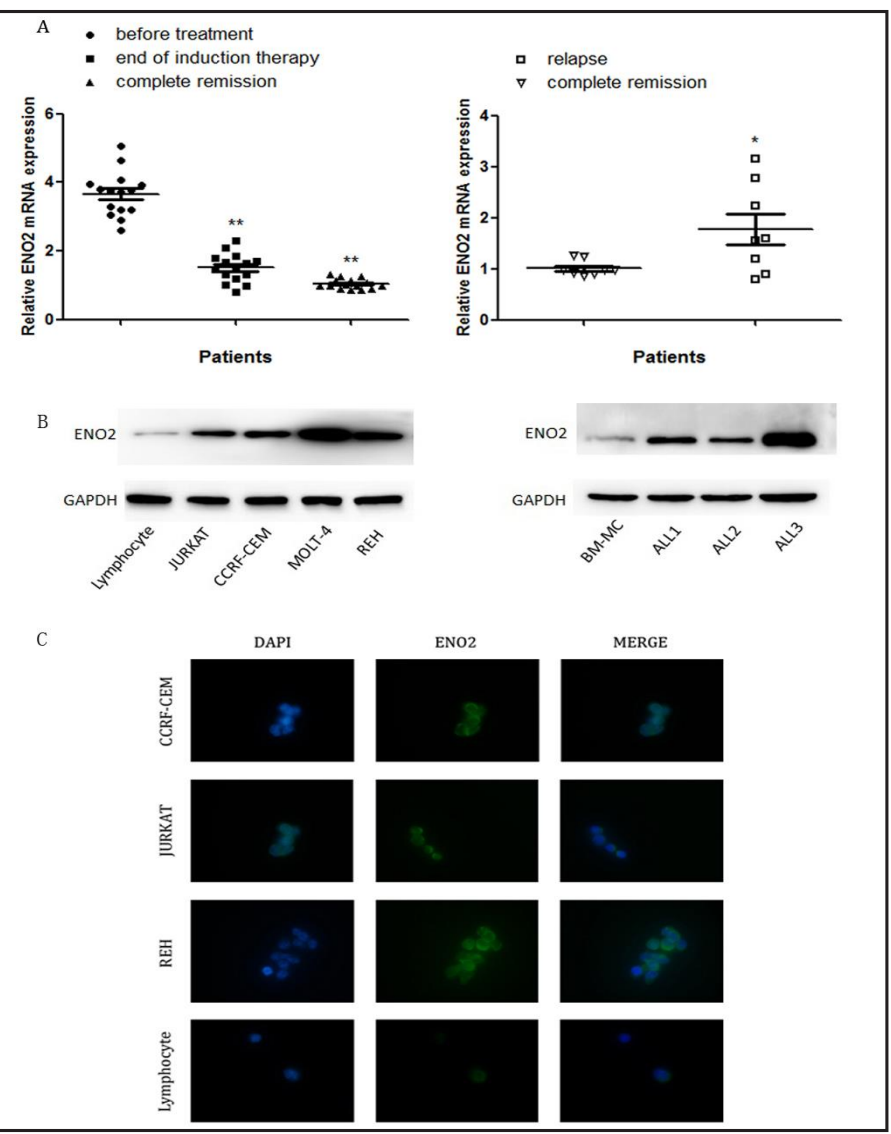

Fig. 1. Expression of ENO2 in ALL patients and ALL cell lines. A: ENO2 mRNA expression of ALL patients during chemotherapy. ENO2 expression was decreased when patients completed induction chemotherapy $\left({ }^{* *} \mathrm{P}<0.01, \mathrm{n}=15\right)$, or reached complete remission $\left({ }^{* *} \mathrm{P}<0.01, \mathrm{n}=15\right)$. In contrast, ENO2 expression was increased when patients suffered relapse $\left({ }^{*} \mathrm{P}<0.05, \mathrm{n}=8\right)$. B: Western blot analysis of ENO2 expression in 4 ALL cell lines and 3 ALL patients. Expression of GAPDH was used as a loading control. C: The location of ENO2 protein in ALL cells, which was detected by immunofluorescence. 
ENO2 promotes clone formation in vitro, and tumorigenicity in vivo

Colony formation assays showed that suppressing ENO2 significantly inhibited cell proliferation compared to sh-Control cells (Fig. 3A). To confirm the effect of ENO2 in ALL cell growth and survival, we analysed the effects of ENO2 silencing on tumorigenicity in vivo using ALL xenograft mouse model. In the experimental group, ENO2-silenced Reh cells were subcutaneously injected into the NOD/SCID mice, and shControl Reh cells were subcutaneously injected into the control group. Visible tumors developed at the injection sites after 7 days. As shown in the growth curve, ENO2-silencing markedly suppressed tumor growth (Fig. 3B). At 35 days, the tumors were removed and weighed (Fig. 3C). ENO2 silencing effectively inhibited the tumor volumes (Fig. 3B) and tumor weights (Fig. 3D) compared to the control group $(\mathrm{P}<0.05)$. However, ENO2 silencing did not significantly affect the body weights of the mice. Immunohistochemistry verified the expression of ENO2 in the shControl-xenografted tumors compared with the reduced expression in shENO2xenografted tumors (Fig. 3E). These results suggested a significant inhibitory effect of decreased ENO2 on tumorigenesis in vivo. In contrast, colony formation assays showed that ENO2 overexpression significantly promoted cell proliferation in vitro and tumorigenicity in vivo compared to control cells (Fig. 3F, $3 \mathrm{G}, 3 \mathrm{H}, 3 \mathrm{I})$.

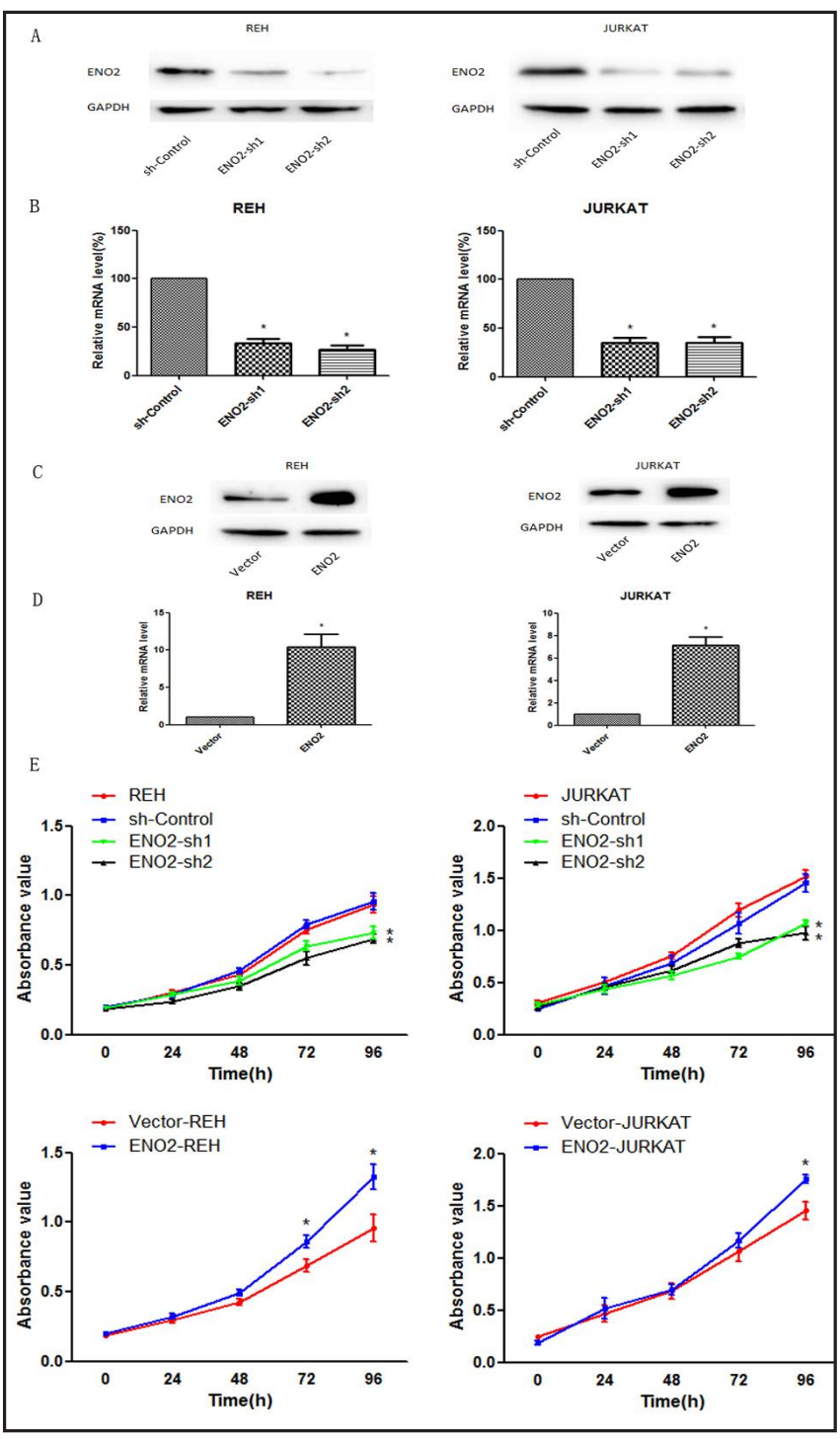

Fig. 2. ENO2 promotes the proliferation of ALL cells. A: The expression levels of protein of ENO2 were confirmed by Western blot after establishing ENO2 silencing ALL cells. B: The expression levels of mRNA of ENO2 were confirmed by real-time RT-PCR after establishing ENO2 silencing ALL cells ( $\mathrm{P}<0.05$, compared with sh-Control group). C: The expression levels of protein of ENO2 were confirmed by Western blot after establishing ENO2 overexpressing ALL cells. D: The expression levels of mRNA of ENO2 were confirmed by real-time RT-PCR after establishing ENO2 overexpressing ALL cells $\left({ }^{*} \mathrm{P}<0.05\right.$, compared with Vector group). E: Cell proliferation of each group cells was monitored over time by CCK- 8 assays $\left({ }^{*} \mathrm{P}<0.05\right.$, compared with sh-Control group).

\section{ENO2-overexpressed up-regulates the glycolysis in ALL cells}

We examined the consumption of glucose in ENO2-silenced ALL cell lines. A significant decrease in glucose consumption, on average $63.8 \%$ of control group in Reh cells and $79.2 \%$ of control group in Jurkat cells (Fig. 4A). Further, we analysed the consumption of glucose 
in ENO2-overexpressing ALL cell lines. A significant increase in glucose consumption, on average $126.5 \%$ of control group in Reh cells and $130.3 \%$ of control group in Jurkat cells, and both can be reduced by 2-DG (Fig. 4B). This finding suggested that ENO2 might up-regulate glycolysis in ALL cells to rescue these cells from apoptosis. It had been reported that ALL cells might increase glucose consumption to prevent glucocorticoid-induced apoptosis; thus, we investigated the sensitivity of glucocorticoid in different ALL cell lines. In the presence of dexamethasone, incubation of leukemic cells with both shENO2 and 2-DG concomitantly, sensitized to dexamethasone compared to sh-ENO2 or 2-DG alone. Co-treatment with sh-ENO2 and 2-DG synergistically inhibited leukemia cell survival by $31.4 \%$ $46.7 \%$, compared to sh-ENO2 or 2-DG alone in Reh cells (Fig. 4C). The same effect was observed in Jurkat cells. The synergism of ENO2-silencing and 2-DG and dexamethasone was best observed in intermediate responsive cells. Highly glucocorticoid-resistant cell line will most likely need higher amounts of drugs to demonstrate an effect. Overall, these data indicate that ENO2-overexpressing cells upregulate glycolysis, and targeting glycolysis in ENO2-overexpressing cells inhibits leukemic survival and concomitantly reverses dexamethasone resistance.

ENO2 promoted cell proliferation, glycolysis, and glucocorticoid-resistance through activation of GSK-3 $\beta$

The overexpression of ENO2 significantly increased the mRNA levels of GLU1, LDH $\alpha$, and PKM2 in ALL cells (Fig. 5A). These genes play important roles in glycolysis in ALL cells. We examined the

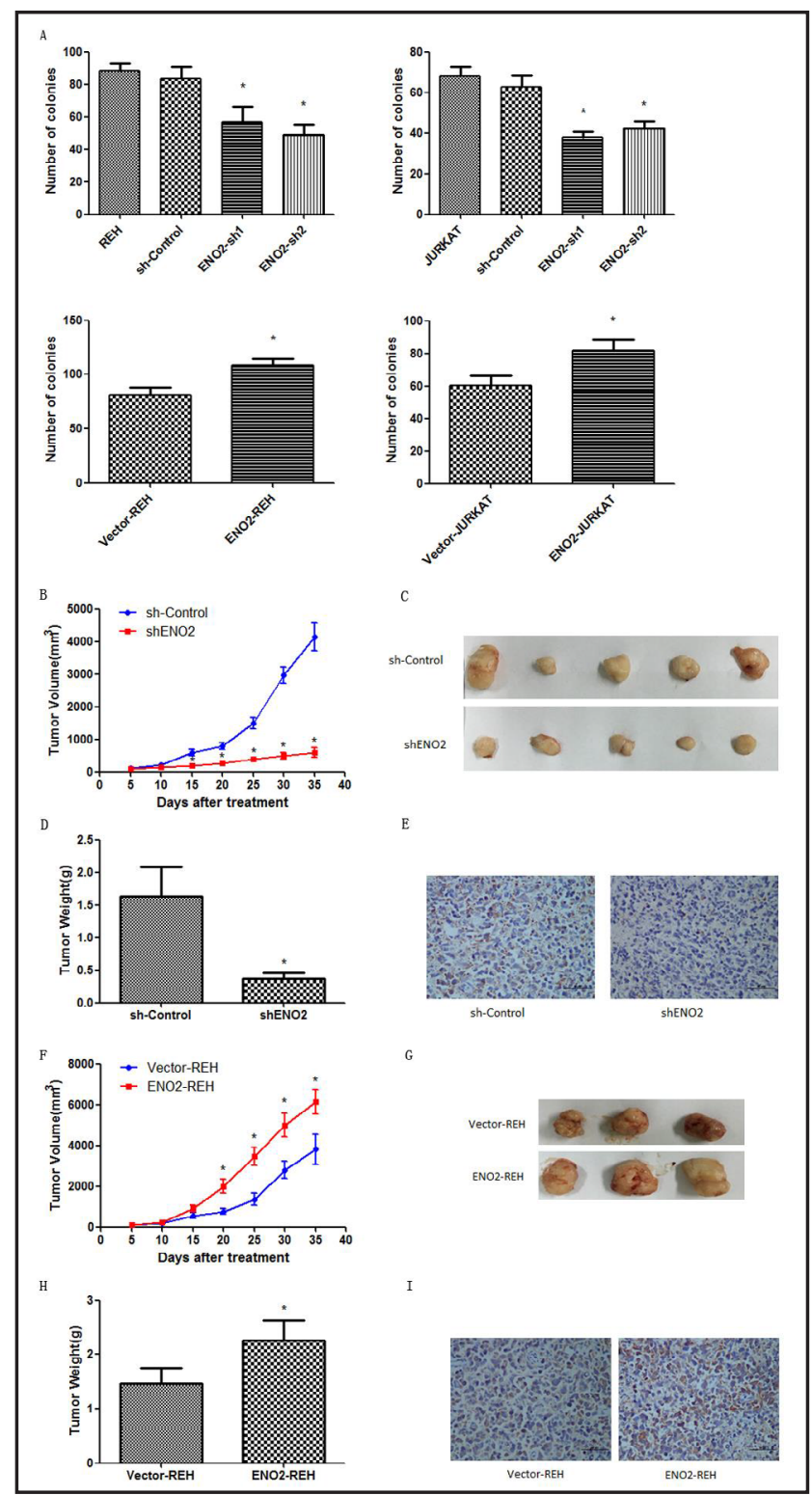

Fig. 3. ENO2 has strong oncogenic function. A: The number of colonies of each group was monitored by colony formation assays $\left({ }^{*} \mathrm{P}<0.05\right.$, compared with sh-Control group). $\mathrm{B}$ : The tumor volume of sh-ENO2 and sh-Control groups was monitored over time (Reh cells) $\left({ }^{*} \mathrm{P}<0.05\right.$, compared with sh-Control group). C: The Images of the xenograft tumors of sh-ENO2 and sh-Control groups formed in NOD/SCID mice. D: The tumor weight of sh-ENO2 and sh-Control groups $\left({ }^{*} \mathrm{P}<0.05\right.$, compared with sh-Control group). E: Immunohistochemical (IHC) staining of ENO2 expression in xenograft tumors. F: The tumor volume of Vector-REH and ENO2-REH groups $\left({ }^{*} \mathrm{P}<0.05\right.$, compared with Vector group). G: The Images of the xenograft tumors formed in NOD/SCID mice. H: The tumor weight of Vector-REH and ENO2-REH groups $\left({ }^{*} \mathrm{P}<0.05\right.$, compared with Vector group). I: Immunohistochemical (IHC) staining of ENO2 expression in xenograft tumors. 
Fig. 4. ENO2 silencing together with 2-DG synergistically sensitizes ALL cells to glucocorticoids. A: The glucose consumption of each group cells $\quad\left({ }^{*} \mathrm{P}<0.05\right.$, compared with sh-Control group). B: The glucose consumption of cells treated with 2-DG $\left({ }^{*} \mathrm{P}<0.05\right)$. C: Leukemia cell survival rate of each group cells after exposure of dexamethasone $\left({ }^{*} \mathrm{P}<0.05\right)$.

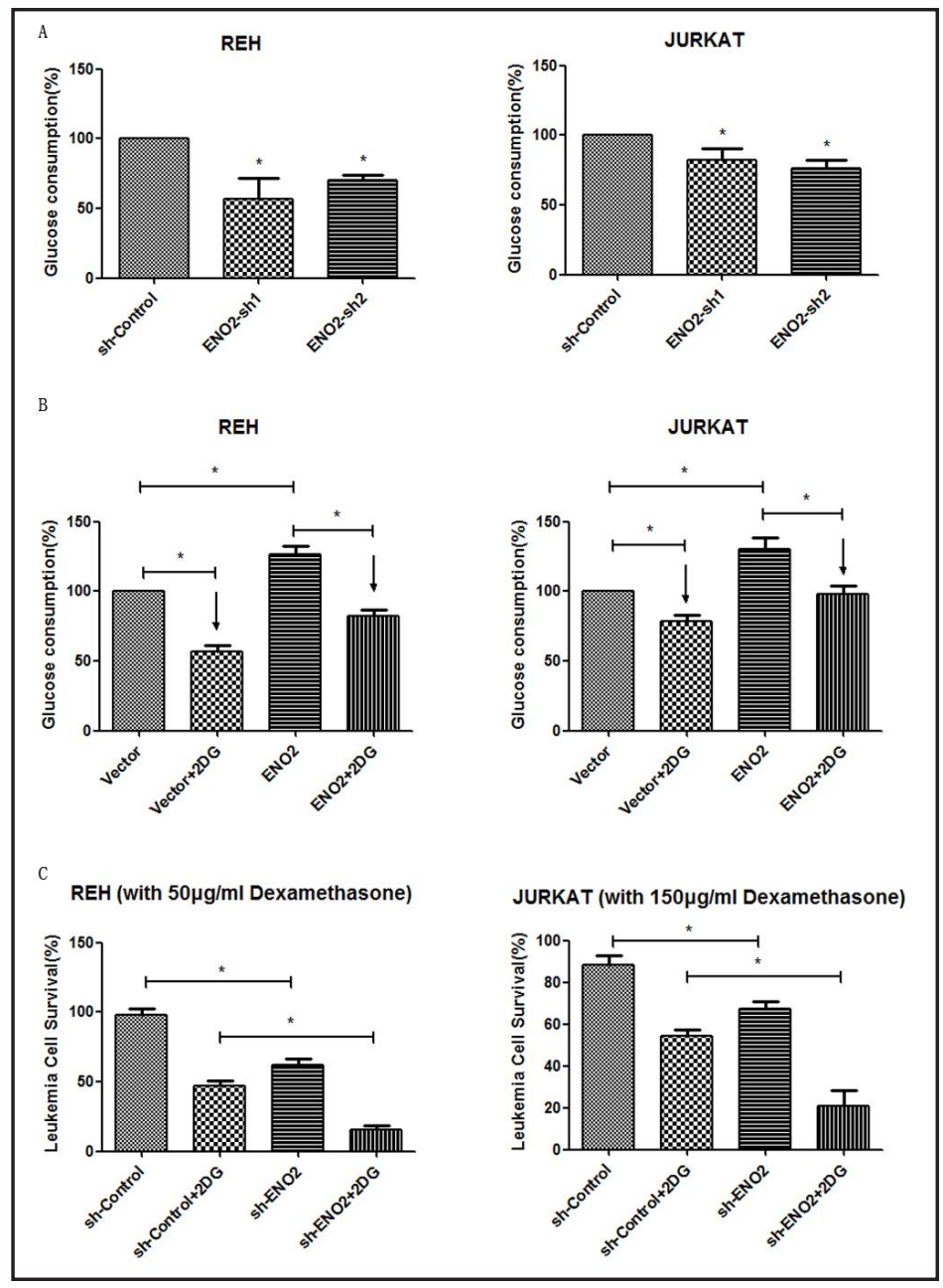

Fig. 5. ENO2 activates the Akt/GSK-3 $\beta$ pathway to promote cell proliferation and glycolysis. A: The mRNA levels of glycolysis related genes of each group cells $\left({ }^{*} \mathrm{P}<0.05\right.$, compared with control group). B: The expression of Akt, phosphorylated Akt, phosphorylated GSK-3 $\beta$ proteins of each group cells.

expression of several proteins involved in the Akt/GSK-3 $\beta$ signal pathway by Western blot analysis and observed the increased expression of phosphorylated Akt and phosphorylated GSK-3 $\beta$ (at Ser-9) in ENO2-overexpressing ALL cells (Fig. 5B). As expected, the Akt/GSK-3 $\beta$ signalling pathway, which plays an important role in cell proliferation and metabolism, was obviously promoted. Notably, the levels of $p$-Akt and p- GSK- $3 \beta$ were dramatically decreased in ENO2-overexpressing ALL cells treated with 2-DG (Fig. 5B). Moreover, 2-DG could reverse the effects of ENO2 on cell proliferation,

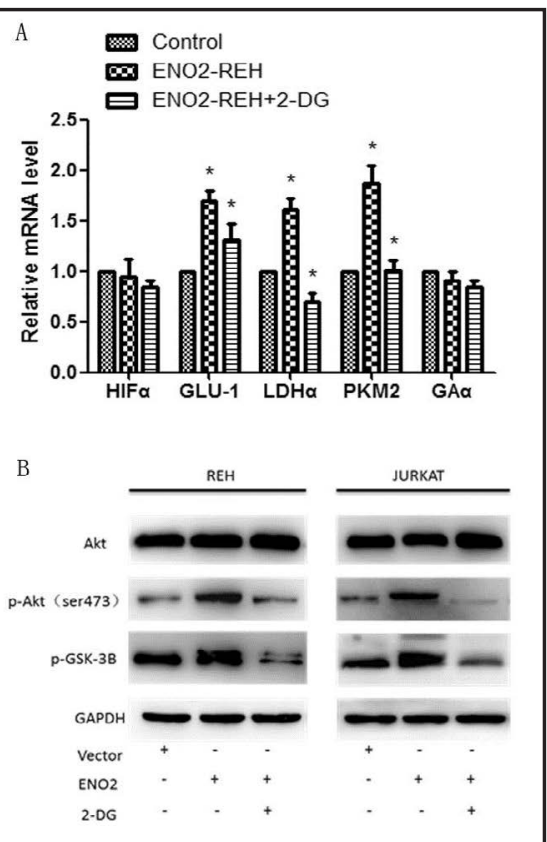


glycolysis, and glucocorticoid-resistance. These results implied that the promotion of cell proliferation, glycolysis, and glucocorticoid-resistance through ENO2 depended on the Akt/ GSK-3 $\beta$ signalling pathway in ALL.

\section{Discussion}

Acute lymphoblastic leukemia is the most common malignant disease in children. ALL patient with a molecular phenotype that was considered to be poor prognostic factor easily lead to drug resistance, or relapse after chemotherapy. Clinical trials for these patients have slightly improved the cure rate of this disease $[11,12]$. Ph/BCR-ABL was known as a high-risk prognostic factor, but the emergence of tyrosine kinase inhibitors has significantly improved CR rates and the outcome of Ph-positive ALL patients [13,14]. Some genes, such as LKB1, PTEN and PI3K/AKT and RAS, have been implicated in regulating the energy metabolism or as part of the process of glycolysis $[15,16]$. These genes may be the potential therapeutic targets. ENO2, also an important gene in the glycolysis of tumor, may provide new insights into the targeted therapy of tumors.

In the present study, the ENO2 mRNA expression levels of patients decreased after chemotherapy. When patients suffered recurrence, the ENO2 mRNA expression levels increased again. This finding could be of great value in the detection of efficacy and recurrence during treatment. Certainly, more cases and data are needed to confirm the relationship among the expression level of ENO2 mRNA, the response to treatment and the relapse.

To explore the potential role of ENO2 in treatment of ALL, we studied the effect of ENO2 on leukemia cell survival and found that ENO2 had strong tumorigenicity, with the overexpression of ENO2 promoting cell growth, tumor formation in NOD/SCID mice and up-regulating the ALL cells glycolysis, which is closely associated with glucocorticoid resistance. Silencing of ENO2 significantly inhibited proliferation and sensitizes ALL cells to glucocorticoids. ENO2 has been proposed to act as a pro-survival factor in cancer cells [17] and supports glioblastoma cell adaptation to cellular stresses, such as serum starvation, hypoxia, chemotherapy and radiotherapy. However, no specific mechanism has yet been proposed [18]. The C-terminal end of ENO2 was shown to promote survival, differentiation and regeneration by activating signal transduction pathways in neuronal cells. The C-terminal end of gamma-enolase contains a PDZ-binding motif, which might enable interactions with several proteins involved in the intracellular redistribution of molecules and signalling pathway events $[19,20]$. In the present study, ENO2 might promote cell growth through the C-terminal end of gamma-enolase, and additional studies are needed to clarify this mechanism.

Considering the poor prognosis of glucocorticoid resistance and the importance of prednisolone and dexamethasone in contemporary ALL treatment protocols, it is vital to develop new therapeutic strategies to reverse glucocorticoid resistance, and these strategies could have a profound impact on ALL treatment efficacy.

Cancer cells, including leukemic cells, utilize higher levels of glucose than normal cells in the presence of oxygen, with an associated increase in lactate production. The phenomenon of aerobic glycolysis, termed the Warburg effect [21-23], involves an increased glycolysis rate due to the up-regulation of several genes involved in glycolysis or glucose uptake $[24,25]$. In the present study, Akt has been implicated in the regulation of glucose uptake and has been shown to induce the expression of glycolysis-related genes, including GLUT-1, LDH $\alpha$, and PKM2. Moreover, the activation of Akt has been shown to specifically activate glycolysis without affecting mitochondrial oxidative phosphorylation [26, 27]. Considering the function of Akt in cell survival, the role of Akt in glucocorticoid resistance is more reasonable. Akt promotes mitochondrial integrity and inhibits cytochrome c release, thereby preventing apoptosis [28-30]. Furthermore, Akt requires glucose to perform its anti-apoptotic functions, further emphasizing the importance of the glycolysis pathway as a target for cancer treatment.

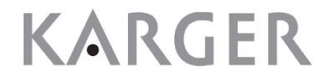


In the present study, the activation of Akt inhibited GSK3 $\beta$ activity and subsequently promoted cell growth and glycolysis. These data indicated that the expression of phosphorylated Akt increased or decreased accordingly when ENO2 was overexpressed or silenced, respectively. This change was positively correlated with the expression level of phosphorylated GSK-3 $\beta$ (inactive form). Taken together, these results suggest that the role of ENO2 in promoting glycolysis is to activate the AKT-GSK3 $\beta$ signalling pathway.

In past years, targeting glycolysis has become increasingly more attractive as a therapeutic approach for several kinds of tumors [31]. The short-term inhibition of glycolysis was not sufficient to exert a significant anti-tumor effect on the body. However, in the present study, a combination of glycolytic inhibitor 2-DG and silencing ENO2 presented a satisfactory result. This synergistic effect reduced the ability of cells to repair damage, which might be a new promising therapeutic approach.

Given that cancer cells have higher glycolytic rates than normal cells, the inhibition of glycolysis more specifically affects tumor cells and has little or no effect on normal cells [32]. In addition, the inhibition of glycolysis did not increase the sensitivity in cells that were already sensitive to glucocorticoids. Several recent clinical trials of glycolytic inhibitors in the treatment of breast, ovarian, lung cancer and malignant gliomas have been conducted [3335]. The present results showed the importance of the glycolytic pathway in glucocorticoid resistance, and targeting the glycolytic pathway may be an alternative approach to reverse glucocorticoid resistance in ALL patients, or in patients for whom many chemotherapy regimens had failed.

\section{Conclusion}

The results of the present study showed that ENO2 expression could be utilized as a gene marker to predict the clinical efficacy of chemotherapy and recurrence. ALL patients with ENO2 overexpression were more likely resistant to glucocorticoids. ENO2 regulated the proliferation and glycolysis of leukemia cells. The regulation of glycolysis pathways associated with ENO2 is a new strategy for the treatment of ALL. In summary, the present study confirmed the important pathobiological role of ENO2 in ALL, but there are still no drugs available for targeting ENO2. Therefore, developing new small molecule inhibitor or antibody is impending, and these strategies may provide a potential therapeutic option for ALL.

\section{Acknowledgements}

Our work was supported by National Natural Science Foundation of China, contract/ grant numbers 81272620 . We thank all the investigators, including the physicians, nurses, pathologists, and laboratory technicians in this study.

This study was approved by the Institutional Review Board of Sun Yat-sen University Cancer Center, and written informed consent was obtained from every healthy volunteer and patient prior to treatment. Additionally, this study was conducted in accordance with the Declaration of Helsinki.

\section{Disclosure Statement}

The authors have declared no conflicts of interest. 


\section{Cellular Physiology Cell Physiol Biochem 2018;46:1525-1535 \begin{tabular}{ll|l} 
and Biochemistry & $\begin{array}{l}\text { DOI: 10.1159/000489196 } \\
\text { Published online: April 24, } 2018\end{array}$ & $\begin{array}{l}\text { @ } 2018 \text { The Author(s). Published by S. Karger AG, Basel } \\
\text { www.karger.com/cpb }\end{array}$ \\
\hline
\end{tabular}}

Liu et al.: Role of ENO2 in Acute Lymphoblastic Leukemia

\section{References}

1 Paul S, Kantarjian H, Jabbour EJ: Adult Acute Lymphoblastic Leukemia. Mayo Clin Proc 2016;91:1645-1666.

-2 Al Ustwani O, Gupta N, Bakhribah H, Griffiths E, Wang E, Wetzler M: Clinical updates in adult acute lymphoblastic leukemia. Crit Rev Oncol Hematol 2016;99:189-199.

-3 Pui CH, Robison LL, Look AT: Acute lymphoblastic leukaemia. Lancet 2008;371:1030-1043.

-4 Oriol A, Vives S, Hernandez-Rivas JM, Tormo M, Heras I, Rivas C, Bethencourt C, Moscardo F, Bueno J, Grande C, del Potro E, Guardia R, Brunet S, Bergua J, Bernal T, Moreno MJ, Calvo C, Bastida P, Feliu E, Ribera JM, Programa Espanol de Tratamiento en Hematologia G: Outcome after relapse of acute lymphoblastic leukemia in adult patients included in four consecutive risk-adapted trials by the PETHEMA Study Group. Haematologica 2010;95:589-596.

-5 Rytting ME, Jabbour EJ, O’Brien SM, Kantarjian HM: Acute lymphoblastic leukemia in adolescents and young adults. Cancer 2017;123:2398-2403.

6 Yokota T, Kanakura Y: Genetic abnormalities associated with acute lymphoblastic leukemia. Cancer Sci 2016;107:721-725.

7 Chiarini F, Lonetti A, Evangelisti C, Buontempo F, Orsini E, Evangelisti C, Cappellini A, Neri LM, McCubrey JA, Martelli AM: Advances in understanding the acute lymphoblastic leukemia bone marrow microenvironment: From biology to therapeutic targeting. Biochim Biophys Acta 2016;1863:449-463.

8 Pui CH, Carroll WL, Meshinchi S, Arceci RJ: Biology, risk stratification, and therapy of pediatric acute leukemias: an update. J Clin Oncol 2011;29:551-565.

-9 Isgro MA, Bottoni P, Scatena R: Neuron-Specific Enolase as a Biomarker: Biochemical and Clinical Aspects. Adv Exp Med Biol 2015;867:125-143.

10 Vizin T, Kos J: Gamma-enolase: a well-known tumour marker, with a less-known role in cancer. Radiol Oncol 2015;49:217-226.

-11 Collins-Underwood JR, Mullighan CG: Genomic profiling of high-risk acute lymphoblastic leukemia. Leukemia 2010;24:1676-1685.

12 Mullighan CG, Downing JR: Genome-wide profiling of genetic alterations in acute lymphoblastic leukemia: recent insights and future directions. Leukemia 2009;23:1209-1218.

13 Mi JQ, Wang X, Yao Y, Lu HJ, Jiang XX, Zhou JF, Wang JH, Jiao B, Shen SH, Tang JY, Gu LJ, Jiang H, Ma LY, Hao SG, Chen FY, Xiong SM, Shen ZX, Chen Z, Chen B, Chen SJ: Newly diagnosed acute lymphoblastic leukemia in China (II): prognosis related to genetic abnormalities in a series of 1091 cases. Leukemia 2012;26:15071516.

14 Mizuta S, Matsuo K, Yagasaki F, Yujiri T, Hatta Y, Kimura Y, Ueda Y, Kanamori H, Usui N, Akiyama H, Miyazaki Y, Ohtake S, Atsuta Y, Sakamaki H, Kawa K, Morishima Y, Ohnishi K, Naoe T, Ohno R: Pre-transplant imatinib-based therapy improves the outcome of allogeneic hematopoietic stem cell transplantation for BCR-ABL-positive acute lymphoblastic leukemia. Leukemia 2011;25:41-47.

15 Vander Heiden MG: Targeting cancer metabolism: a therapeutic window opens. Nat Rev Drug Discov 2011;10:671-684.

16 Tissing WJ, den Boer ML, Meijerink JP, Menezes RX, Swagemakers S, van der Spek PJ, Sallan SE, Armstrong SA, Pieters R: Genomewide identification of prednisolone-responsive genes in acute lymphoblastic leukemia cells. Blood 2007;109:3929-3935.

17 Takei N, Kondo J, Nagaike K, Ohsawa K, Kato K, Kohsaka S: Neuronal survival factor from bovine brain is identical to neuron-specific enolase. J Neurochem 1991;57:1178-1184.

-18 Yan T, Skaftnesmo KO, Leiss L, Sleire L, Wang J, Li X, Enger PO: Neuronal markers are expressed in human gliomas and NSE knockdown sensitizes glioblastoma cells to radiotherapy and temozolomide. BMC Cancer 2011;11:524.

19 Hafner A, Glavan G, Obermajer N, Zivin M, Schliebs R, Kos J: Neuroprotective role of gamma-enolase in microglia in a mouse model of Alzheimer's disease is regulated by cathepsin X. Aging Cell 2013;12:604614.

20 Ponting CP, Phillips C, Davies KE, Blake DJ: PDZ domains: targeting signalling molecules to submembranous sites. Bioessays 1997;19:469-479.

-21 Bhattacharya B, Mohd Omar MF, Soong R: The Warburg effect and drug resistance. Br J Pharmacol 2016;173:970-979. 


\section{Cellular Physiology Cell Physiol Biochem 2018;46:1525-1535 \begin{tabular}{l|l} 
DOI: 10.1159/000489196 & $\begin{array}{l}\text { O 2018 The Author(s). Published by S. Karger AG, Basel } \\
\text { www.karger.com/cpb }\end{array}$
\end{tabular}}

Liu et al.: Role of ENO2 in Acute Lymphoblastic Leukemia

22 Lu J, Tan M, Cai Q: The Warburg effect in tumor progression: mitochondrial oxidative metabolism as an anti-metastasis mechanism. Cancer Lett 2015;356:156-164.

-23 Upadhyay M, Samal J, Kandpal M, Singh OV, Vivekanandan P: The Warburg effect: insights from the past decade. Pharmacol Ther 2013;137:318-330.

-24 DeBerardinis RJ, Mancuso A, Daikhin E, Nissim I, Yudkoff M, Wehrli S, Thompson CB: Beyond aerobic glycolysis: transformed cells can engage in glutamine metabolism that exceeds the requirement for protein and nucleotide synthesis. Proc Natl Acad Sci U S A 2007;104:19345-19350.

-25 Buentke E, Nordstrom A, Lin H, Bjorklund AC, Laane E, Harada M, Lu L, Tegnebratt T, Stone-Elander S, Heyman M, Soderhall S, Porwit A, Ostenson CG, Shoshan M, Tamm KP, Grander D: Glucocorticoid-induced cell death is mediated through reduced glucose metabolism in lymphoid leukemia cells. Blood Cancer J 2011;1:e31.

26 Ito K, Suda T: Metabolic requirements for the maintenance of self-renewing stem cells. Nat Rev Mol Cell Biol 2014;15:243-256.

27 Wenner CE: Targeting mitochondria as a therapeutic target in cancer. J Cell Physiol 2012;227:450-456.

28 Jacobs SR, Herman CE, Maciver NJ, Wofford JA, Wieman HL, Hammen JJ, Rathmell JC: Glucose uptake is limiting in T cell activation and requires CD28-mediated Akt-dependent and independent pathways. J Immunol 2008;180:4476-4486.

29 DeBerardinis RJ, Lum JJ, Hatzivassiliou G, Thompson CB: The biology of cancer: metabolic reprogramming fuels cell growth and proliferation. Cell Metab 2008;7:11-20.

-30 Coloff JL, Mason EF, Altman BJ, Gerriets VA, Liu T, Nichols AN, Zhao Y, Wofford JA, Jacobs SR, Ilkayeva O, Garrison SP, Zambetti GP, Rathmell JC: Akt requires glucose metabolism to suppress puma expression and prevent apoptosis of leukemic T cells. J Biol Chem 2011;286:5921-5933.

-31 Vander Heiden MG: Exploiting tumor metabolism: challenges for clinical translation. J Clin Invest 2013;123:3648-3651.

32 Vander Heiden MG, Cantley LC, Thompson CB: Understanding the Warburg effect: the metabolic requirements of cell proliferation. Science 2009;324:1029-1033.

33 Pelicano H, Martin DS, Xu RH, Huang P: Glycolysis inhibition for anticancer treatment. Oncogene 2006;25:4633-4646.

34 Oudard S, Carpentier A, Banu E, Fauchon F, Celerier D, Poupon MF, Dutrillaux B, Andrieu JM, Delattre JY: Phase II study of lonidamine and diazepam in the treatment of recurrent glioblastoma multiforme. J Neurooncol 2003;63:81-86.

35 Raez LE, Rosenblatt J, Schlesselman J, Langmuir V, Tidmarsh G, Rochalima C, Papadopoulos K, O'Connor J, Baldie P, Lampidis T: Combining glycolytic inhibitors with chemotherapy: Phase I trial of 2-deoxyglucose and docetaxel in patients with solid tumors. Journal of Clinical Oncology 2005;23:238S. 